UCRL- 97661

PREPRINT

CONF-880408--10

\title{
Optical Imaging Diagnostics for Fusion Plasmas
}

UCRL --97661

\author{
S. I. Allen
}

DE88 008682

This paper was prepared for submittal to the Proceedings of the Matcrials Research Socicty Conference

April 5-9, 1988, Reno, Nevada

April 1, 1988

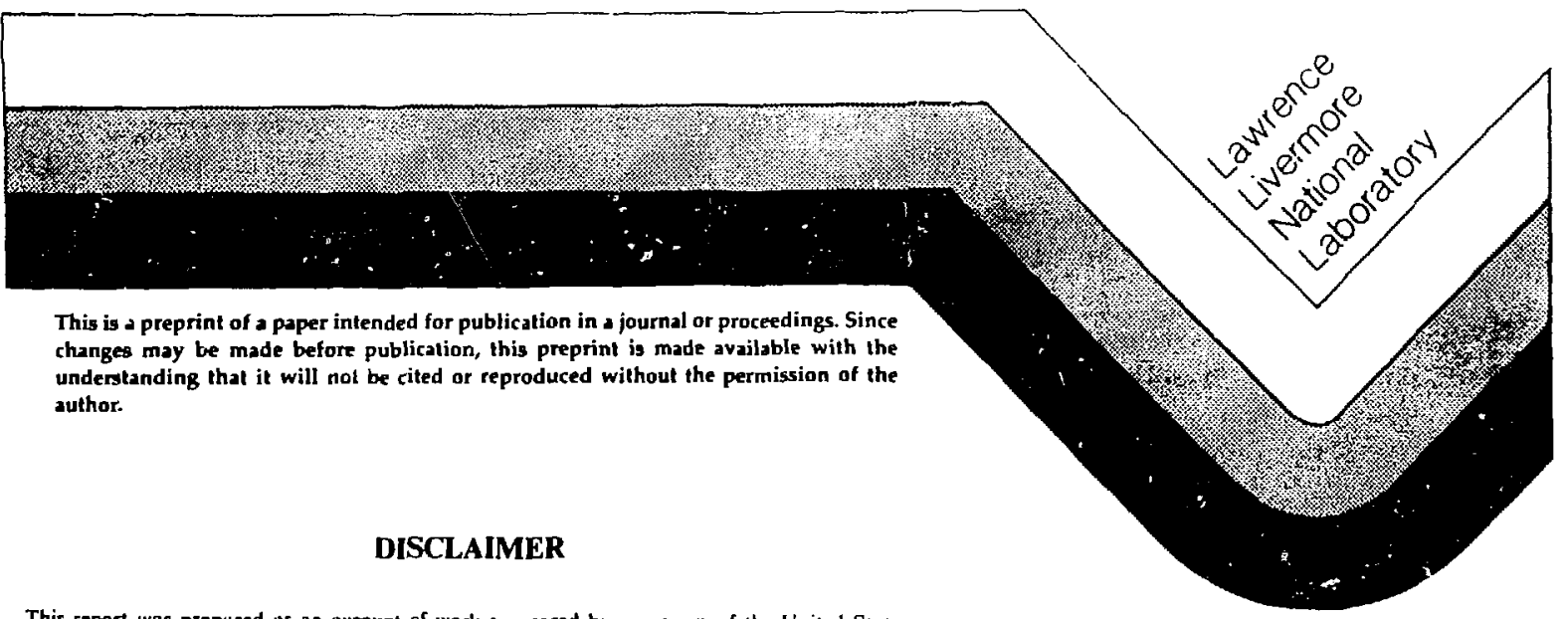

This report was prepared as an account of work spunsored by an agency of the United States Government. Neither the United States Government nor any agency thereof, nor any of their employees, makes any warranty, express or implied, or assumes any legal liability or responsibility for the jccuracy, completeness, or usefulness of any information, apparalus, product, or process disclused, or represents that its use would not infringe privalely uwned rights. Reference herein to any specific commercial product, process, or service hy trade name, trademark. manufacturer, or otherwise does not necessarily constitute or imply its endursement, recommendation, or favoring by the United States Guvernment or any agency therenf The vieus

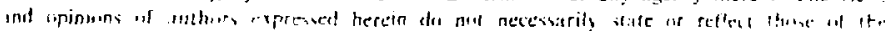




\title{
OPTICAL IMAGING DIAGNOSTICS FOR FUSION PLASMAS
}

\author{
S. L. ALLEN, Lawrence Livermore National Laboratory, Livermore, C'A 94550
}

\begin{abstract}
Inaging diagnostics are used for spatially -and temporally-resolved quantitative measurements of plasma properties such as the ionization particle source, particle and energy loss, and inpurity radiation in magnetically confined fusion plasmas. Diagnostics equipped with multi-element solid-state detect ors (often with inage intensifiers) are well suited to the enviromment of large fusion machines with high magnetic fields and $x$-ray and neitron fluxes. We have used both conventional (16 ms/frame) and highspeed viden cameras to measure neutral deuterium $\mathrm{H}_{\alpha}(6563 \AA)$ emissions from fusion plasmas. Continuous high-speed measurements are made with video cameras operat ing at $0.1-0.5 \mathrm{~ms} /$ frame; gated cameras provide snapshots of $10-100 \mu \mathrm{s}$ during each 16-ms video frame. Digital data acquisition and absolute intensity calibrations of the cameras enable detailed quantitative source measurements; these are extremely jumportant in determining the particle balance of the plasma. In a linear confinement device, radial transport : an be deternined from the total particle balance. In a toroidal confinement devirs, the details of particle recycling can be determined. Optical imaging in other regions of the spectrum are also important, particularly for the divertor region of large tokamaks. Absolutely calibrated infrared cameras have been used to image the temperature changes in the walls and thereby determine the heat flux. Absolutely calibrated imaging ultraviolet spectrometers measure impurity concentrations; both spatial and spectral imaging instruments are employed. Representative data from each of these diagnostic systems will be presented.
\end{abstract}

\section{INTRODUCTION}

Complex plrenomena in inagnetically confined fusion plasmas require time-resolved measurements at many locations. Early diagnostics used multiple discrete channels or shot-by-shot spatial scanning to obtain one-dimensional spatial profiles. Advances in solid-state imaging detectors have made it possible to obtain time-resolved twodimensional images of the plasma. Sophisticated electronic readout systems have been developed for these cameras so that extremely high time resolution (2000 to 12000 frames/s) is possible. Image intensifiers can be coupled to the imaging detectors to increase their sensitivity; gating the intensifier enables snapshots of $10-100 \mu$ s to be obtained. Image converters also can be coupled to the cameras so that they are sensitive at ultraviolet and $\mathrm{x}$-ray wavelengths. Detailed analysis of these data has been made possible by the development of complicated image-processing techniques.

These cameras are extremely useful for qualitative measurements of plasma properties, but they also can be used to obtain detailed, quantitative results. We discuss here the use of quantitative video measurements on two types of nagnetic fusion devices. The first application is on a linear magnetic confinement device-the tandem mirror machine 


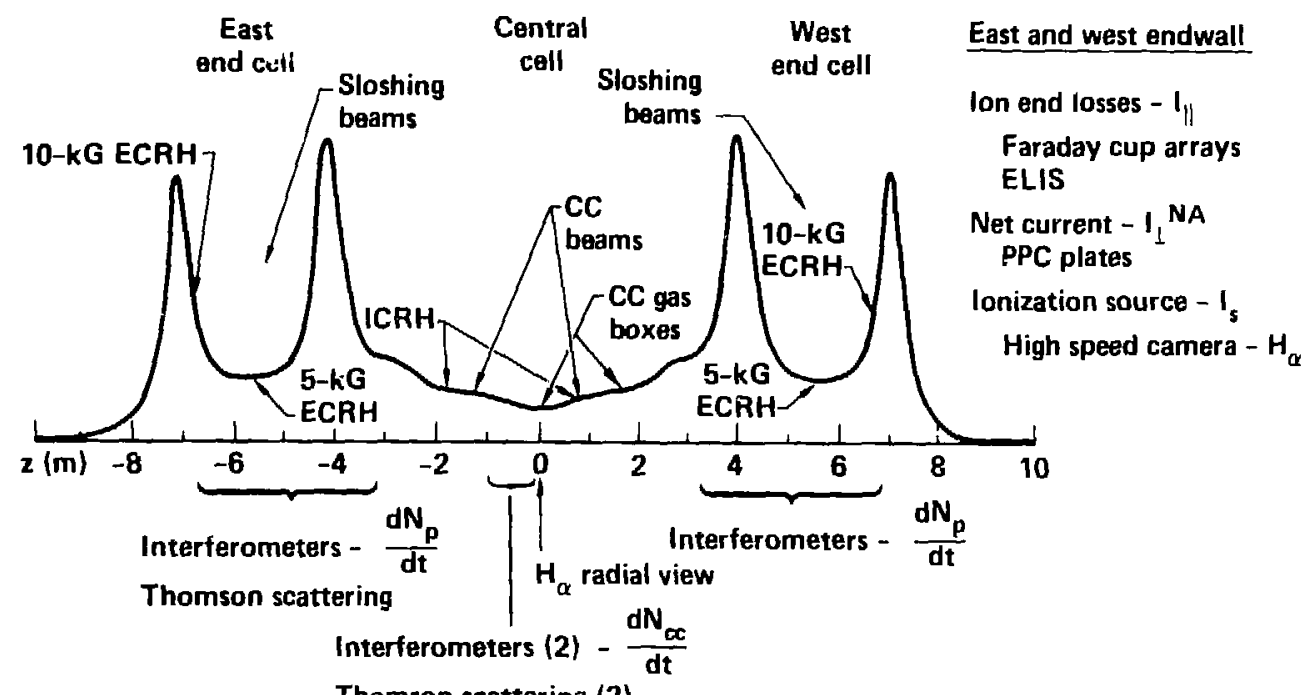

Thomson scattering (2)

Figure 1: Magnetic field profile for $T M X-U$. Located at each end wall are diagnostics to measure the particle balance.

[1] (TMX-U) --at Lawrence Livermore National Laboratory, Livermore, California. In this application, the video data are used to obtain a two-dimensional measurement of the ionization in the plasma and to thereby determine the particle source for comparison with the measured particle losses. The emphasis here will be on the details of the diagnostic measurements; further details can be found in Ref. [5]. The second application is on a toroidal confinement device-the DII]-D tokamak [2]--at General Atomics, San Diego, California. In this experiment, video data are used to study the neutral particle transport in the edge of the plasma and to measure the heat loads on the walls of the vessel. Further details of these neasurements can be found in Ref. [6]. In each application, the video data are only one part of a large set of integrated diagnostic measurements. The relevant physics and associated diagnostic instruments are briefly described for each application.

\section{PARTICLE SOURCE MEASUREMENTS WITH VIDEO DATA}

Quantitative particle source measurements have been carried out with video data on the TMX-U experiment. A detailed explanation of the operation of this machine is outside the scope of the present discussion and is presented elsewhere $[3,4]$. For reference, a schematic of the magnetic field profile is shown in Fig. 1. Briefly, TMX-U uses a combination of electron-cyclotron resonance heating (ECRH) in the end cells and neutral-beam injection in both the end cells and the central cell. The plasma is circular in the middle of the central cell, with a diameter of $25 \mathrm{~cm}$, and is elliptical at the locations of the magnetic field peaks (i.e., magnetic flux is conserved). The overall length of the machine is about $20 \mathrm{~m}$, as shown in Fig. 1. Plasma confinement is achieved by a combination of the magnetic field and the plasma potential. Plasma 


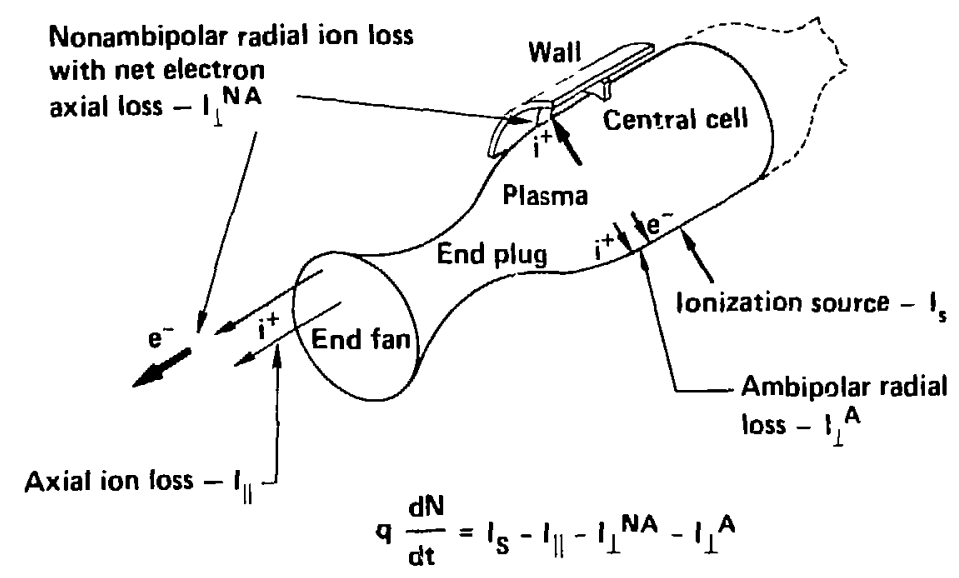

Figure 2: Particle sources and losses in a tandem mirror plasma. Most particle losses are measured by electrical currents. The ionization source is mensured by a fast video camera, allowing us to estimate the (ambipolar) radial transport that does not have an associated electrical current.

fueling is accomplished by gas puffing in the central-cell region (labeled gas box in the Fig. 1).

For the present discussion, it is important to note that the linear plasma column in TMX-U has a core electron density in the $10^{12} \mathrm{~cm}^{-3}$ range, measured by microwave interferometers, and a core clectron temperature of about $100 \mathrm{eV}$, measured by Thomson scattering. The length of the each plasma pulse is $100 \mathrm{~ms}$, limited by the duration of the heating systems. The plasma is mostly hydrogen or deuterium, as the measured impurity densities are low. The major goals of this device are to confine particles and energy so that efficient fusion reactions can occur; we will focus here on particle confinement. Because of the inherently open-field geometry of the tandem mirror, most plasma losses can be measured directly by means of electrical currents. The particle sources and losses are shown schematically in Fig. 2. Specifically, the particle losses are divided into axial and radial losses:

1. Ions that are lost axially out the ends of the machine are denoted by $\mathbf{I}_{\|}$. This ion current is measured by a set of Faraday cup collectors equipped with $-3-\mathrm{kV}$ grids to repel electrons. A special end-loss ion spectrometer (ELIS) also measures the ion current as a function of mass and energy on the axis of the machine.

2. Ions that are lost radially out of the machine can be accompanied by an axial electron loss current denoted by $\mathrm{I}_{\perp}^{\mathrm{NA}}$. This is called a nonambipolar radial loss and the net axial electron loss current is measured by a set. of segmented plates at each end of the machine. 
3. Ions that are lost radially out of the machine aiso can be accompanied by an equal radiul elcut ron loss current. This so-called ambipolar radial loss IA does not produce a net clectrical current .

The ambipolar radial ion loss $\mathrm{I}^{\mathrm{A}}$ could be measured by a series of gridded analyzers (to sweep out electrons) placed to measure radial losses. However, this would require a large number of detectors placed very close to the plasma. A serond approach is to use the particle balance equation to detemine the ambipolar loss $I^{A}$ :

$$
q \frac{d \mathrm{~N}}{d^{+}}=\mathrm{l}_{\mathrm{S}}-\mathrm{l}_{\|} \quad \mathrm{l}_{\perp}^{\mathrm{NA}}-\mathrm{I}^{\mathrm{A}},
$$

where $\mathrm{q} \mathrm{dN} / \mathrm{dt}$ is the buildup of particles in the plasma and $l_{\mathrm{S}}$ is the plasma source. (Particle flows in this equation are denoted by particle currents.) The tern $\mathrm{q} d \mathrm{~N} / \mathrm{dt}$ is measured by microwave interferometers placed at several locations in the machine. By measuring $l_{S}$, we can determine the term $I^{A}$ in the plasma. Because the source $I_{S}$ is the result of gas puffing and has a complicated, three-dimensional spatial dependence, its measurement is ideally suited to video techniques. In addition, vidco cameras are much easier to align than discrete detectors, which is particularly important in the complicated geonet ry of TMX- $\mathrm{C}^{\mathrm{S}}$.

\section{Camera Measurements of the Ionization Source}

The term $I_{S}$ is obtained from absolutely calibrated two-dimensional camera measurements. Figure 3 shows a cross section of 'ТМX-U and the locations of the cameras: one camera (end view) views the plasma parallel to the plasma column from the end of the machine; the other camera (side view) views the plasma from the side of the plasma column near the gas box. The end-view camera capilaljzes on the linear geometry of the TMX-U device: the view antomatically integrates the emission along the plasma column so that the total emission as a function of radius is obtained. In practice, this integration could be limited by the depth of field of the optical system, so it was verified experinentally that the depth of field was greater than the length of the machine. The side-view camera measures the details of the axial distribution of the emission near the gas box, mainly for detailed comparisons with computer models of neutral particle transport. The camera system and digitizer are identical to those used for the tokamak experiments, so they will be described in detail in the next section concerned with measurements on the DIII-D tokamak.

All end-view measurements were made with a Spin Physics SP-2000 camera system [7]. The camera is a solid-state sensor with 240 by 192 pixels, and the resulting spatial resolution is about $1.0 \mathrm{~cm}$. An integral tape drive and digitizer system acquires the data. The ultimate (full-frame) rate of the camera is 2000 frames/s; partial frames can be acquired six times faster. For most data on TMX-U, 1-ms time resolution was used during the 100-ms plasma shot. The camera head is mounted on the end of TMX-U and is subjected to magnetic fields (hundreds of gauss), $x$-ray bursts, and electrical noise from all of the heating systems. For most operation, no major interference is measured. The fast tape drive is located $100 \mathrm{ft}$ from the camera head, behind a shield wall. A fiber optic link connects the camera unit with a Hewlett-Packard 9920 microcomputer in the control room. The microcomputer automatically rewinds the tape, digitizes each of the (100) frames desired, and stores the data between shots. (Note that data compaction is very important in this application because the camera acquires the equivalent of 5-10 


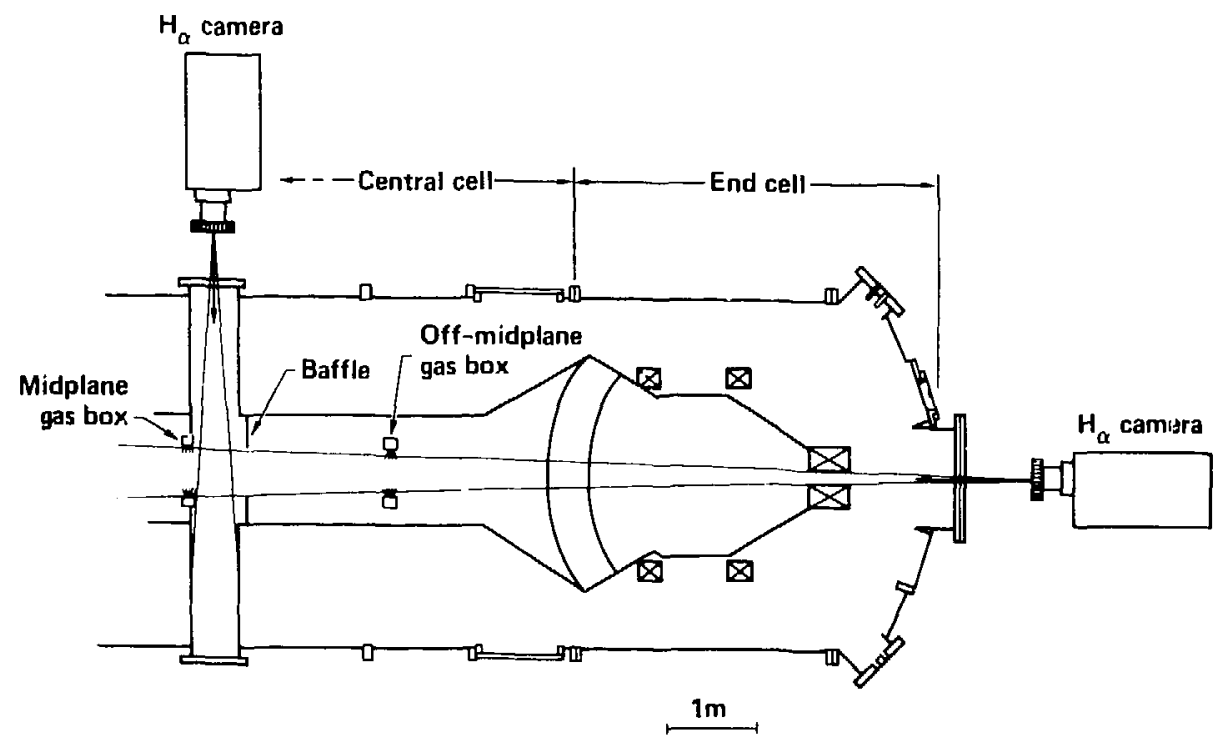

Figure 3: Locations of video cameras on TMX-U. The end-view camera automatically obtains the axial integral of the emission.

MBytes of data on tape for each shot; the computer stores only about 0.5 MByte.) The data are finally sent to the main data computer for comparison with the other particle loss terms. The camera is triggered several seconds before the shot so the tape drive can come to full speed about $1 \mathrm{~s}$ before the shot. A calibrated timing-triggering system is used to synchronize the camera with the plasma shot so that the time of each camera frame is known accurately for comparison with other diagnostics.

The actual determination of $I_{S}$ is based on a measurement of the visible emission from the Balmer-alpha $\left(\mathrm{H}_{\alpha}-6561 \AA\right)$ line of atomic neutral hydrogen (or deuterium) in the plasma. Detailed atomic physics calculations [8] have been made to determine the branching ratio, $R$, between the ionization and excitation of the neutral atom. For $T_{e}$ greater than about $20 \mathrm{eV}$, and $n_{e}$ in the $10^{12} \mathrm{~cm}^{-3}$ range, $R$ is relatively insensitive to $T_{e}$ and is equal to about 11 ionizations/photon. These are the relevant parameters for the core of the TMX-U plasma. At the plasma edge, where the electron density and temperature are lower, $\mathrm{R}$ is sensitive to the electron temperature. In addition, molecular hydrogen is present in this region and contributes to the ionization. A Monte Carlo computer code of neutral transport, called DEGAS [9], equipped with atomic physics models indicates that $\mathrm{R}$ can be as large as $25-30$ at the plasma edge. Therefore, the most reliable measurements of $I_{S}$ are in the plasma core, defined to be $\mathbf{r} \leq 10 \mathrm{~cm}$.

The optical system of the camera is equipped with an interference filter having a 30$\AA$ bandpass centered at $6561 \AA$. Thereforc, the camera is effectively a "two-dimensional spectrometer," measuring only the $\mathrm{H}_{\alpha}$ emissions from the plasma. The bandpass of the filter is chosen narrow enough so that other impurity lines are not measured, but wide enough to match the collimation of the optical system so that the interference filter does not cause a spatially dependent response (i.e., incoming rays are nearly parallel to the filter). A sample of several frames of $\mathrm{H}_{\alpha}$ video data are shown in Fig. 4. 


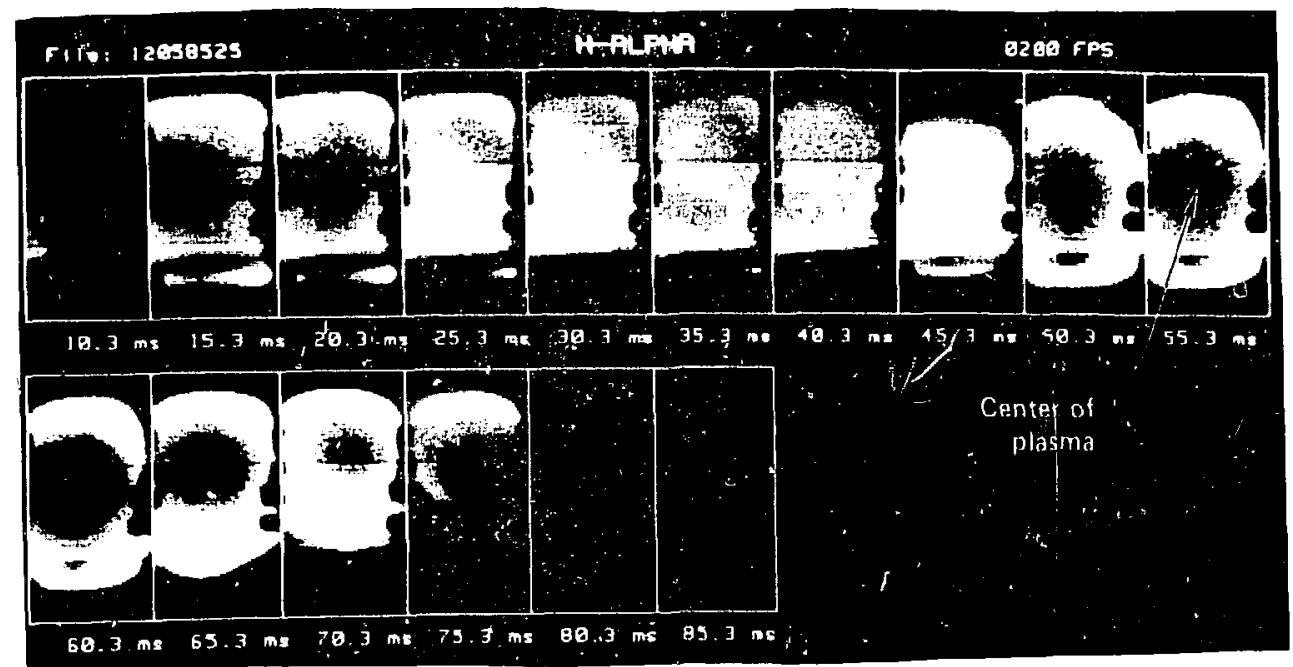

Figure 4: Several frames of data from the end-view camera.

The camera system was calibrated using techniques traceable to the Nationa! Bureau of Standards (NBS) [10]. The exact optical system used in the experiment was used to view an NBS-calibrated tungsten ribbon lamp (the bright ness as a function of wavelength is specified by NBS). Estimated uncertaint ies in this calibration are about 10-15\%. The camera system was also checked carefully for linearity. As experted, it was found to be linear up to a certain signal level, followed by a compression in the response to expand the dynamic range. Pixel-to-pixel uniformity was also checked and found to be small enough that corrections were not necessary.

An example of the comparison of $I_{S}$ with the other terms in the particle balance is shown in Fig. 5. For these data, the core $\mathrm{H}_{\alpha}$ emission has been integrated and multiplied by the alomic physics factor $R$ to obtain $I_{5}$ as a function of time. The other curve shows all of the terms in Eq. 1 (each is the total current for the core plasma $\mathrm{r} \leq$ $10 \mathrm{~cm}$ ) except for $I^{A}$, i.e., the difference between the two curves is $I^{A}$. (Note that the plasma conditions for this special test are that $I_{\|}$is the largest loss.) The agreement between the $I_{S}$ curve from the video daia and the other terms shows that $I^{A}$ is small for these conditions. The $I_{S}$ measurements were checked under various plasma conditions, and for most cases, $I^{A}$ was small. However, conditions with large $I^{A}$ were discovered. In addition, the two-dinensional video measurements allowed us to check the particle balance as a function of radius. In general, we found that $\mathrm{I}^{\mathrm{A}}$ increased at the plasma edge. (Additional details can be found in Ref. 5.) These video measurements were the first systematic determination of ambipolar transport in a tandem mirror. In addition, they enabled detailed studies of neutral transport, fueling efficiency, and gross plasma stability (i.e., plasma motion). The comparisons of the data with the DEGAS neutral transport computer code are particularly important but are outside the scope of the 


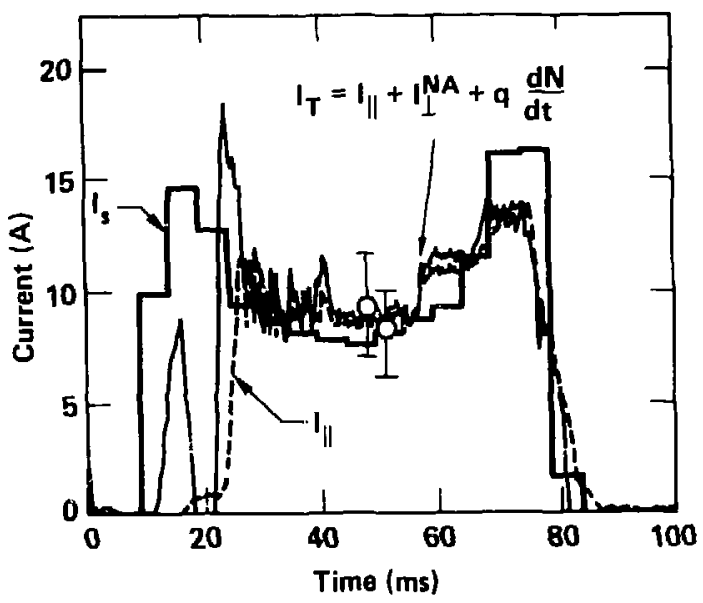

12/5/85-S4

Figure 5: A comparison of the measurement of $\mathrm{I}_{5}$ with the other terms in the particle balance. For this shot, $\mathrm{I}_{\mathrm{S}}$ equals $\mathrm{I}_{\mathrm{T}}$, signifying that the residual ambipolar transport $\mathrm{I}^{\mathrm{A}}$ is small.

present discussion. However, this code is even more important for tokamak studies; therefore, we will discuss the edge plasma measurements on the DIII-D tokanak and briefly compare these data with DEGAS.

\section{CAMERA MEASUREMENTS ON THE DIII-D TOKAMAK}

The DIII-D tokamak is a toroidal magnetic confinement device with a noncircular cross section. The core of the plasma has closed flux surfaces. Outside this region are open field lines that intersect the plasma wall; this region is called a magnetic divertor. The core plasma has an electron temperature of several keV, and the electron density ranges between $10^{13}$ and $10^{14} \mathrm{~cm}^{-3}$. The edge plasma is similar to the core of the tandem mirror plasma, in that the $T_{e}$ is $\simeq 50 \mathrm{eV}$ and $n_{\mathrm{e}}$ is $\simeq 10^{13} \mathrm{~cm}^{-3}$. The DIII-D plasma cross section is shown in Fig. 6 with the diagnostic system used to measure the edge plasma.

Because of the 4-s duration of the DIII-D plasma pulse, conventional video data rates ( $16 \mathrm{~ms} /$ frame) are often adequate, so CCD cameras such as the SONY-XC38 are used, again equipped with $\mathrm{H}_{\alpha}$ interference filters. These cameras are mounted inside the magnetic field coils of the tokamak and experience large, changing magnetic fields (several hundred gauss) and electrical noise. Under most conditions, the camera image is not affected. However, under some plosma conditions, there are large fluxes of highenergy $x$ rays (tens of $\mathrm{keV}$ ) and neutrons. These cause the image field to be filled with white spots, presumably because of direct interaction of the $x$ ray or neutron flux with the CCD. In some cases of extreme neutron flux, slight permanent damage to the CCD las been observed. The optical systems are carefully adjusted to keep the cameras 


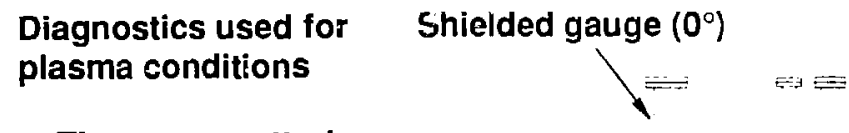

- Thomson scattering

- $\mathrm{CO}_{2}$ interferometer

- ECE

- Magnetic probes

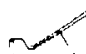

Divertor IR camera $\left(165^{\circ}\right)$
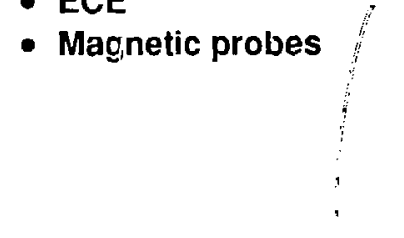

\section{Divertor Langmuir probes $\left(180^{\circ}\right)$}
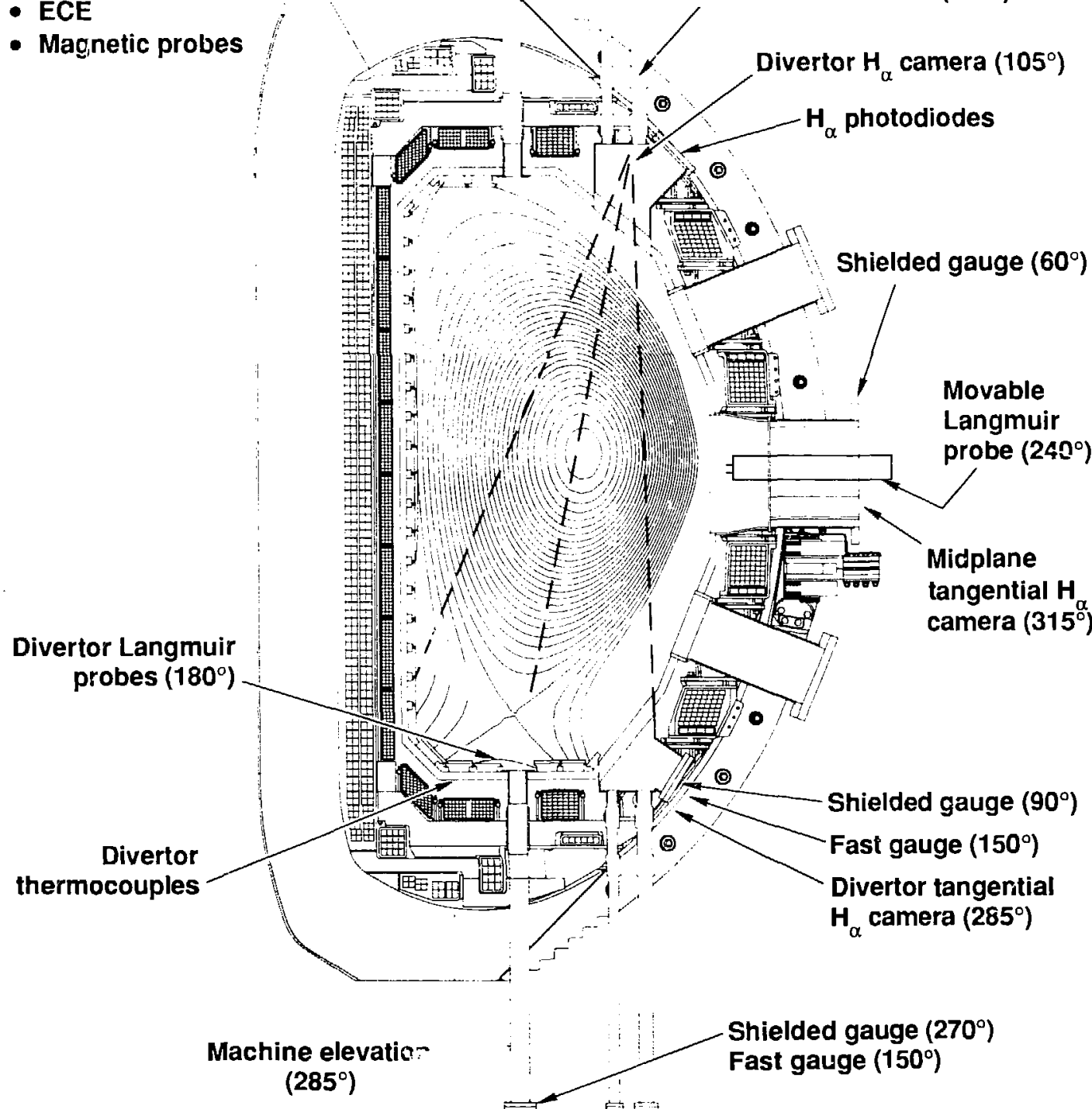

(1)

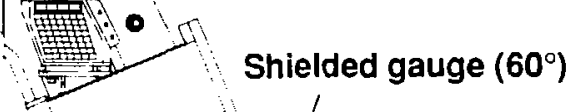

Machine elevatio? $\left(285^{\circ}\right)$

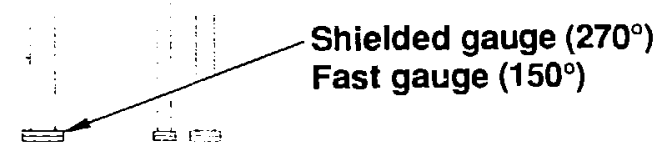

Figure 6: Cross section of the DIII-D tokamak showing the noncircular cross section and magnetic divertor. The video cameras view the plasma from above; the diagnostics used to measure the edge plasma are also indicated. 


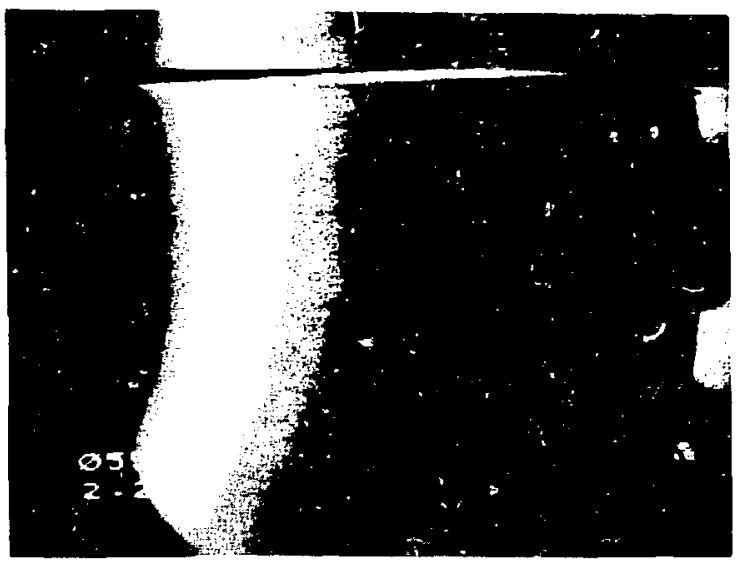

Figure 7: A single frame of video data from the $H_{\alpha}$ camera on Dill-D; the two bands are the strike points of the plaswa on the wall

in the linear range and to keep the signal levels below the threshold of the camera's automatic gain control. The intensity calibration technique is similar to that discussed in the previous section.

Because of the large amount of data acquired during the 4-s plasma shot, the data are acquired by two systems: a commercial-grade editing tape recorder, and a custom video-digitizing system. The videotape storage ailows detailed analysis of each frame by digitizing the data after the shot. However, as shown by the sample video frame in Fig. 7, the data are quite toroidally symmetric.

A custom electronics system [11] was developed to take a radial cut of each video field (a horizontal line in the middle of Fig. 7) in real time. Only the radial slice is digitized by a CAMAC module, with 312 points/slice (a radial resolution of about $1 \mathrm{~cm}$ ). An HP Series 300 microcomputer then acquires, stores, and processes the data from the CAMAC crate. The computer can stack these video slices so that the time history of the $\mathrm{H}_{a}$ emission in the divertor region can be studied; an example is presented in Fig. 8 . Note the very dark period starting at about $1.9 \mathrm{~s}$. This marked drop in the $\mathbf{H}_{a}$ emission occurs soon aiter the neutral beams are turned on to heat the plasma. The stored energy of the plasma increases, and the energy confineinent time also increases. The new confinement mode is usually called $\mathrm{H}$-mode, for high confinemnent. The large bursts of emission after this period are called edge-localized modes, or FLMs; the radial profile of $n_{e}$ and $T_{\varepsilon}$ change dramatically during this time. We also have used a camera with a gated image intensifier (XYBION) to study these rapid bursts. With this camera, the whole image does not saturate on very bright bursts; instead, only the part of tne image that is bright has a noninear response. This camera is ideally suited to our DIII-D 


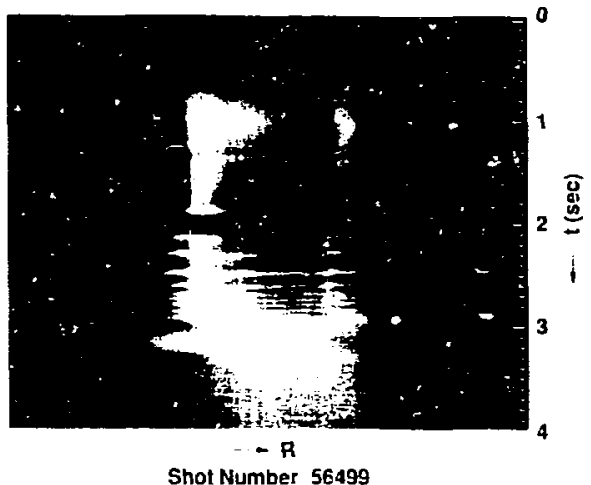

Figure 8: The result of real-time data compression and pracessing. The $H_{\alpha}$ emission is shown as a function of radius (horizontal) and iime (vertical).

application, where the image has very bright and dark regions. A detailed analysis of $\mathrm{H}$ mode behavior and ELMs is outside the scope of the present discussion and is discussed in Ref. [12].

As discussed, the modeling of the $\mathrm{H}_{\alpha}$ ennission in the divertor region. is very important because most of the plasma ionization source originates there. In the divertor region of DIII-D, the electron temperature is low $(\simeq 10 \mathrm{eV})$, and the $\mathrm{H}_{\alpha}$ emission is particularly sensitive to $T_{e}$. Therefure, we used the DEGAS code to model the $\mathrm{H}_{\alpha}$ emission directly. The diagnostics shown in Fig. 6 measure the plasma parameters required for DEGAS. Specifically,

1. An array of Langmuir probes mounted on the divertor plates measure $n_{e}$ and $T_{e}$ in the divertor region. A movable Langmuir probe at the midplane measures the $\mathrm{n}_{e}$ and $\mathrm{T}_{e}$ edge profiles.

2. An infrared camera at a location that is similar to the $\mathrm{H}_{\alpha}$ camera measures the heat flux to the divertor plates. This camera is absolutely calibrated by viewing thermocouples mounted in the walls. The camera measures a quantity propor- 
3. Several ionization gauges measure the neut ral pressure as a function of position.

An edge plasma model is used to connect these dat a at various positions. The DEGAS code then calculates the neutral density as a function of position, and finally calculates the neutral pressure profile and the actual $\mathrm{H}_{\alpha}$ camera inage. The DEGAS code can be rum in a full three-dinensional mode, but we have used a two-dimensional simplification hecause of toroidal symmetry. DEGAS contains the full geometry of the machine. inchuting wall composition and shape, and has both an atomic physics and wall-interation package (e.g.. wall reflection). The code requires approximately 1 hr of calculations on a Cray supercomputer. The DEGAS code allows for a full correction of the $H_{a}$ emission (i.e.. ile factor $R$ as discussed for tandem mirror plasmas) as a function of prosition.

Comparison of the DEGAS out put with the experimentally measured $H_{\alpha}$ emission has shown agreement to within a factor of two. 'The code can also calculate the neu. iral pressure as a fumction of position, and similar agrcement has been obtained. Most. imporianty. the st udy has shown that a large amount of the nentrals "recycle" in ihe divertor region. That is. only about $10 \%$ of the particle flux in the divertor region actually supplies ionization to the core plasma. Present work is concentrated on obtaining better video data, particularly detailed measurements in the divertor region. A camera equipped with special viewing optics (i.e., a "periscope") has been mounted at the same height as the "X-point" of the magnetic field in Fig. 6. This camera has a tangential view of the plasma and therefore should allow us to measure the divertor region in detail. These measurements are important for detailed measurements of the neutral transport and for comparisons with DEGAS.

\section{SUMMARY}

Optical imaging techniques are valuable in magnetic fusion devices. We have presented only two examples dealing with quantitative measurements: particle confinement studies in a linear tandem mirror machine, and neutral particle transport in the divertor region of a tokamak. These measurements have capitalized on the high time resolution (2000 to 12000 frames/s) and high spatial resolution (several hundred pixels in each dimension) of solid-state cameras. In addition, these camera systems are relatively insensitive to the magnetic fields, electrical noise, $x$ rays, and neutrons encountered on a magnetic fusion device. Digital data-handling techniques, particularly data compaction, make it possible to process these data and compare them with other diagnostic systems.

Many other optical imaging dcvices are used on magnetic fusion devices, such as $\mathrm{x}$-ray imaging cameras [13] and imaging visible spectrometers. In addition, ultraviolet. spectrometers that image in space [14], wavelength [15], or both [16] have been used. Future magnetic fusion devices will be bigger and more complicated, with a large premium on diagnostic access, time resolution, and detailed data acquisition. In short, optical imaging techinques will become even nore important in future devices.

\section{ACKNOWLEDGMENTS}

Work performed under the auspices of the U.S. Department of Energy by Lawrence Livernore National Laboratory under Contract W-7405-Eng-48. 


\section{REFERENCES}

[1] D.P. Grubb, S.L. Allen, T.A. Casper, et al., Phys. Rev. Lett. 53 (1979) 783.

[2] J. Luxon, P. Anderson, F. Batty, et al., in Plasma P!ysics and Controlled Nuclear Fusion Research 1986 (Proc. 11 Int. Conf., Kyoto, Japan), vol. 1, IAEA, Vienna (1987) 159.

[3] T.C. Simonen, S.L. Allen, T.A. Casper, Phys. Rev. Lett. 55 (1983) 1668.

[4] S.L. Alle und the TMX-U Experimental Team, Rev. Sci. Instrum. 56 (1985) 873.

[5] S.L. Allen, D.L. Correll, D.N. Hill, et al., Nucl. Fusion 27 (1987) 2139.

[6] S.L. Allen, to be published in Proc. of Eighth Int. Conf. on Plasma Surface Interactions in Cantrolled Fusion Devices, Jülich, Federal Republic of Germany (1988).

[7] J. Bixby, Proceedings of SPIE 301 (1981).

[8] L.C. Johnson, E. Hinnov, J. Quant. Spec. Rad. Trans. 13 (1973) 333.

[9] D. Heifetz, D Post, M. Petravic, et al., J. Comput. Phys. 46 (1982) 309.

[10] R. Stair, R.G. Jolunston, E.W. Halbach, J. Res. Nat. Bur. Stan. A64 (1960) 291.

[11] S.W. Ferguson, D.K. Kevan, D.N. Hill, S.L. Allen, in 12th Symp. on Fusion Engineering, Monterey, CA, October 12-16, 1987.

[12] K.H. Eurrell et al., Phys. Rev. Lett. 59 (1987) 1432.

[13] R.J. Fonck, K.P. Jaehnig, E.T. Powell, P. Roney, "Tangentially Viewing Soft X-ray Camera for Current Density and Internal Shape Measurements on a Noncircular Tokamak," to be published in Rev. Sci. Instrum.

[14] R.K. Richards, H.W. Moos, S.L. Allen, Rev. Sci. Instrum. 51 (1981) 1.

[15] R.G. Fonck, A.T. Ramsey, R.V. Yelle, Applied Optics 21 (1987) 2115.

[16] D. Content, M. Perry, D. Wroblewski, H.W. Moos, Optical Eng. 26 (1987) 806. 